\title{
The challenge raised by Gaia
}

\author{
Annie C. Robin \\ Observatoire de Besançon, Institut Utinam, Université de Franche-Comté, France \\ email: annie.robin@obs-besancon.fr
}

\begin{abstract}
Gaia will perform an unprecedented high quality survey of the Milky Way. Distances, 3D kinematics, ages and abundances will be obtained, giving access to the overall mass distribution and to the Galactic potential. Gaia data analysis will involve a high level of complexity requiring new and efficient multivariate data analysis methods, improved modelling of the stellar populations and dynamical approaches to the interpretation of the data in terms of the chemical and dynamical evolution of the Galaxy.
\end{abstract}

Keywords. Galaxy: stellar content, Galaxy: evolution, Galaxy: dynamics

The Gaia instruments will perform accurate photometry and astrometry up to magnitude 20 and spectroscopy to 16 th magnitude. The astrometric accuracy is expected to be at the level of 10, 20, $100 \mu$ as for stars at $G=10,15,20$ resp. (Brown, 2008). This astrometric accuracy will permit measurement of parallactic distances up to the Galactic center at the level of $10 \%$. The photometry will be accurate at the level of the $0.2 \mathrm{mmag}$ at $\mathrm{G}=15$ and 2.5 millimag at $\mathrm{G}=20$. From the RVS, the radial velocity errors will be better than $1 \mathrm{~km} \mathrm{~s}^{-1}$ for bright and cool stars, and at the end of the mission $30 \mathrm{~km} \mathrm{~s}^{-1}$ for stars at $V=16$. Gaia will also provide astrophysical parameters from the $\mathrm{BP} / \mathrm{RP}$, such as, for a star at $G=15$, effective temperature at the level of 1 to $5 \%$, gravity to $0.1-0.4$ dex, metallicity to better than 0.2 dex. Extinction will be measured on hot stars from RVS with an accuracy of $0.05-0.1 \mathrm{mag}$. Thus, Gaia provides full characterization for populations to $G=15$ (accurate distance, age, abundances, 3D velocities). Consequently, ages will be determined from the astrophysical parameters and stellar evolution models, as well as relative ages from the elemental abundances. This will enable us to trace the chemo-dynamical evolution of different populations. For example one expects to have about:

- 1.5 billion stars ( $0.5 \%$ of the stellar content of the Galaxy) with photometry, parallaxes and proper motions. Among them, about $9 \times 10^{8}$ stars belonging to the thin disc, $4.3 \times 10^{8}$ to the thick disc, $2.1 \times 10^{7}$ to the spheroid and $1.7 \times 10^{8}$ to the bulge.

- 200 million stars with spectroscopy $(G<16)$ (astrophysical parameters $T_{\text {eff }}, \log g$, $[\mathrm{Fe} / \mathrm{H}]$, radial velocity)

- 6 million stars with elemental abundances $(G<12)$

- Variabilities and binarity.

All populations in the Galaxy will be surveyed. Although a limited number of stars truly in the bulge will be measured with the spectrograph due to extinction and crowding, (Robin et al, 2005), most stars in the bulge region will have their parallaxes and proper motions measured.

In the meantime, complementary surveys are planned that will enhanced the Gaia outputs, among them LSST, RAVE, PanStarrs and JASMINE. All these surveys will efficiently complement Gaia, giving better accuracy on radial velocities for fainter stars, exploring deeper fields, furnishing denser light curves for variables, revealing dusty regions from the near infrared, etc. 
Having data sets for billions of objects, covering large numbers of multi-epoch observables, the data analysis will be a real challenge. The experience with recent large scale surveys will be of some help but previous surveys have never reached this level of complexity. The question is: how to turn Gaia data into a clear understanding of Galactic dynamics and evolution?

A promising path is to use modelling as a tool for analysis, interpretation and confrontation between the data and scenarios of formation and evolution of the Milky Way. Various modelling options are pursued. Since Galactic evolution leaves traces both in the stellar kinematics and the abundances, both aspects have to be taken into account. For this reason, the stellar population-synthesis approach will be valuable, allowing us to compare scenarios of Galaxy formation and evolution with the Gaia data set by simulating catalogues with the same observables and comparable accuracies. The Besançon Galaxy model is such a project (Robin et al, 2003), constrained by already existing surveys like GSC2, DENIS, 2MASS, SDSS, etc., from multi-wavelength data (from X to infrared) and multivariate catalogues (photometric, astrometric and spectroscopic). It produces realistic simulations of the stellar content of the Galaxy with characteristics in agreement with our present knowledge of Galactic evolution and taking into account interstellar extinction. It is already used in Gaia preparation and is planned to be exploited for the data analysis.

Complementary to the synthetic approach, dynamical models allow the reconstruction of the Galactic potential from the space distribution and the kinematics of numerous stars or selected tracers. Several dynamical approaches are being pursued, like the Schwarzshild approach, the torus method (Dehnen \& Binney 1996), N-body simulations or adaptative N-body techniques such as the "Made-to-Measure" scheme (Syer \& Tremaine 1996), or quadratic programming (Dejonghe, 1989). The drawback is that the data sets are biased with parameters generally not included in the dynamical analysis - the first bias being the limiting magnitude but more complex biases also exist, such as those generated by the fact that the accuracy depends on magnitudes, colours and position on the sky, and the bias coming from interstellar extinction. As a consequence, these dynamical modelling approaches will benefit from being coupled with the synthetic approach.

Using such tools for the interpretation of Gaia data will require us to develop efficient methods of multivariate data analysis and model fitting, like genetic algorithms, or Markov Chain Monte Carlo. Inverse methods will be attempted but are not easy to handle with the large parameter space furnished by Gaia.

A huge challenge is then raised by Gaia interpretation. One can expect that no simplistic model will straightforwardly fit the data. Complemented by other surveys, the Gaia catalogue will encompass any model view. Even though imperfect, the modelling will still be useful to i) understand the imperfections of our knowledge, ii) help to interpret the findings, iii) test physical scenarios of Galaxy formation and evolution, iv) describe the dynamics of the system, in relation with the dark matter content, and v) place the scenario of formation in the cosmological context and constraints cosmological models.

\section{References}

Brown, A. G. A. 2008. AIP Conf. Series 1082, 209-215.

Dejonghe, H., 1989, ApJ, 343, 113.

Dehnen, W., Binney, J., 1996, ASP Conference Series, Vol. 92, pp. 393.

Robin, A. C., Reylé, C., Derrière, S., \& Picaud, S. 2003, A\&A, 409, 523

Robin, A. C., Reylé, C., Picaud, S., Schultheis, M. 2005. A\&A430, 129.

Syer, D., Tremaine, S., 1996, MNRAS282, 223. 